\title{
11 marzo 2021: Giornata Mondiale del Rene. "Vivere bene con una malattia renale"
}

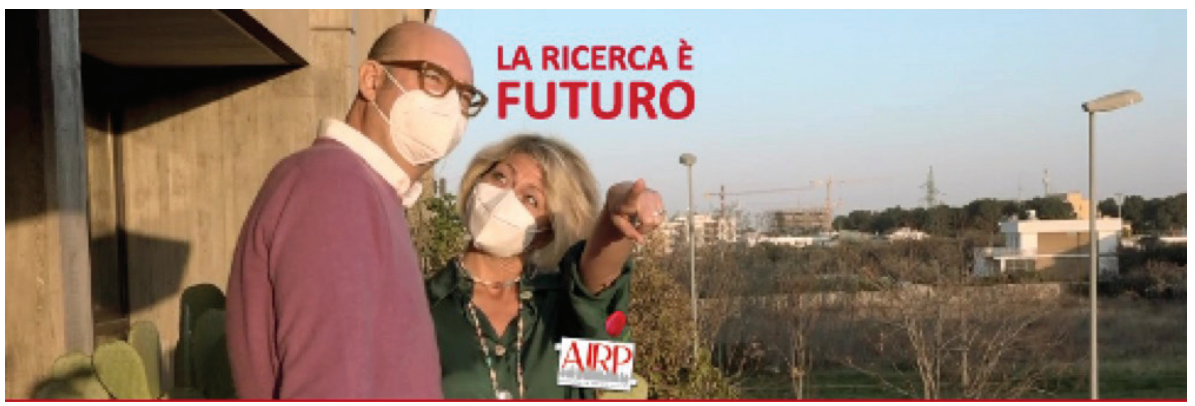

"Dare e ricevere sono la stessa cosa: l'amore che dai resta tuo per sempre"
V.: lo, purtroppo, soffro di rene policistico come tutti gli associati ad AIRP. È qualcosa che ho ereditato dalla nostra famiglia e, quindi, come potrai immaginare, la parola "rene policistico, creatinina, dialisi, trapianto" è qualcosa con cui ho dovuto familiarizzare sin da piccolo. Questa, ovviamente, è una spada di Damocle che ti porti sulla testa da quando sei piccolo e sai benissimo quale sarà l'evoluzione, quindi, da un lato, c'è un voler negare questa chiara evidenza e, dall'altro, arrivi a una sana lucidità rispetto a questo problema e, di conseguenza, conosci bene quali sono le strade. Man mano che l'evoluzione della malattia mi ha portato a un'insufficienza renale cronica, io e Tania ci siamo guardati negli occhi e abbiamo cercato di capire quali potessero essere le strade: la dialisi non era per me un'opzione, perché ho visto così tante persone a me care soffrire e portarsi per tanti anni lunghe sedute di dialisi che hanno deteriorato velocemente il proprio corpo e, quindi, c'era in me il desiderio forte di fare un trapianto. Mia madre ha fatto tre trapianti di rene a partire dai lontani anni '90. Una pioniera, perché li ha fatti all'estero (Belgio), quando in Italia di trapianto si parlava poco e, quindi, di conseguenza, ricordo benissimo come lei tornava rigenerata da questi trapianti e la qualità della vita che aveva dopo.

\section{L.: La parola d'ordine è consapevolezza.}

T.: lo sono entrata "da ospite" in questo mondo. Sono i famosi "mondi invisibili", che solo quando ci capiti inizi a conoscere e poi, una volta dentro, cerchi di trovare un tuo posto. Nonostante noi fossimo consapevoli del viaggio che avevamo intrapreso, la strada è stata più complicata del previsto, perché Vittorio aveva bisogno di prepararsi alla ricezione del rene mediante una nefrectomia bilaterale, a cui è seguito un breve quanto necessario e salvifico periodo di dialisi (sebbene lui sia stato sempre timoroso e poco favorevole rispetto alla stessa, perché ha visto soffrire troppo sua mamma e i familiari che purtroppo ha perso a causa di questa patologia). I suoi occhi sono pieni di tanta sofferenza e tanto dolore e, quindi, si può comprendere questa sua riluttanza. La dialisi sicuramente salva molte persone e la donazione è semplicemente un'altra strada, da considerare, per continuare a vivere.

V.: Sì, la parola d'ordine è "consapevolezza" e la unirei al concetto di informazione, perché oggi la consapevolezza 


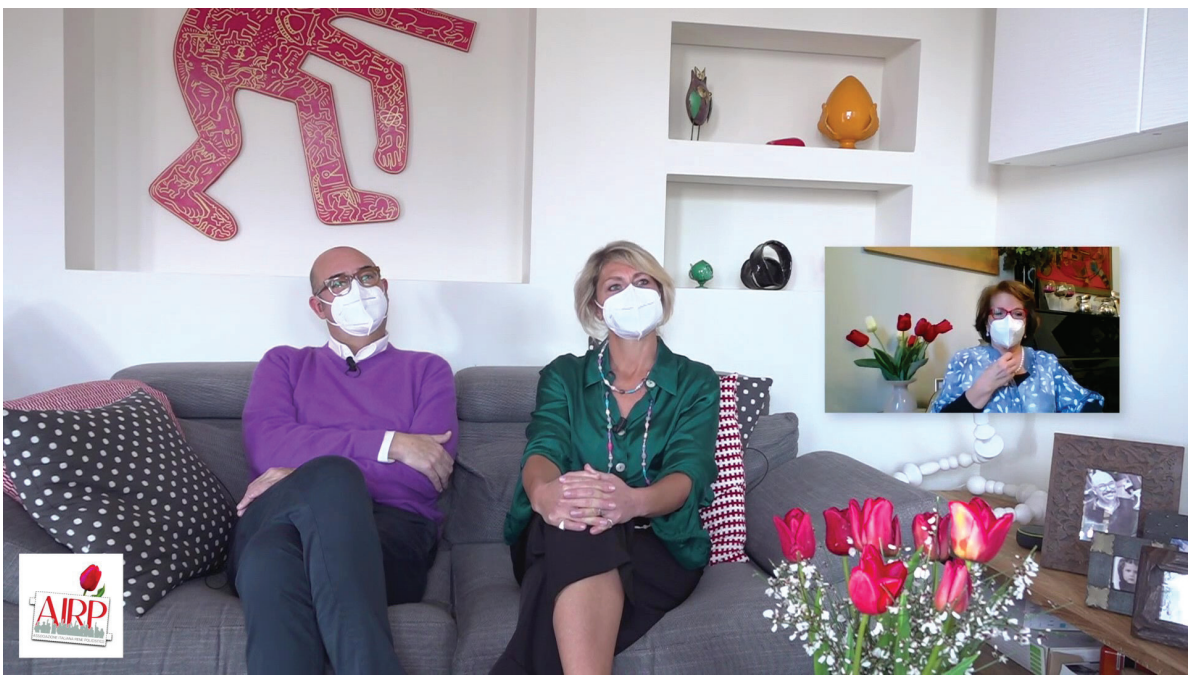

grata a coloro che erano in grado di sostenerlo, affiancarlo e rassicurarlo dove io non ero in grado di farlo, quindi veramente grazie ad AIRP.

\section{L.: AIRP è stato il vostro navigatore satellitare?}

V.: Assolutamente sì. Devo dire che anche la comunità scientifica che fa parte di AIRP è di grosso sostegno. I numerosi workshop e salotti che hai organizzato durante il Covid-19, in maniera virtuale, ci hanno permesso anche di entrare in contatto con personalità italiane importanti, che conoscono bene la nostra patologia e che hanno dato supporto scientifico a

richiede anche un buon livello di conoscenza su quelle che sono le opzioni. È chiaro che avere la certezza di arrivare, a un certo punto, ad avere un rene che non funziona più correttamente presuppone che tu debba trovare una soluzione e, chiaramente, fare una scelta informata è un'esigenza.

\section{L.: Come avete fatto a conoscere AIRP (Associazione Ita-} liana Rene Policistico) inserirei qui visto che poi è citata di nuovo la sigla, come vi siete avvicinati ad AIRP, dove l'avete trovata e come è stato il primo approccio?

V.: Mi sono avvicinato ad AIRP diversi anni fa. Man mano che la consapevolezza che ci fosse la necessità di affrontare il problema, piuttosto che negarlo, come facciamo per tanti anni, mi ha portato a cercare informazioni, e AIRP per me è stata fondamentale perché è una comunità di persone come me, che hanno lo stesso tipo di patologia, che vivono le mie stesse problematiche e con cui ho la possibilità di scambiare un'opinione, di chiedere un consiglio o, talvolta, semplicemente di condividere il mio stato d'animo e ricevere empatia.

T.: lo vorrei dire grazie a te, Luisa, e a chi ha voluto questa comunità. lo credo che in AIRP ci sia una moltitudine di persone, come Vittorio, con il desiderio di trovare un contatto, una connessione umana, delle risposte, il perché stia accadendo una cosa specifica. Risposte che spontaneamente sempre arrivano e che, a volte, io leggevo sui post dell'Associazione, perché ho voluto associarmi anch'io ad AIRP, per comprendere e capire meglio e non fare da mera osservatrice passiva, ma per affiancare Vittorio, anche se nel silenzio. A volte, leggendo dei suoi post, mi dicevo: "Ma non mi aveva raccontato che ultimamente aveva le mani fredde". Io vedevo che in AIRP molte persone rispondevano alle sue domande, persone di certo molto più esperte e qualificate di me, perché ognuno è esperto nella sua patologia e nella problematica che sta vivendo da anni e che ha visto vivere in famiglia. Quindi, io lì educavo me stessa, mi informavo e, attraverso il web, venivo anche a conoscenza di cose che Vittorio non mi aveva ancora detto, senza che ciò mi infastidisse, anzi ero quelle che, a volte, sono cose empiriche che noi viviamo e condividiamo. Sai, Luisa, condividere le proprie sensazioni, dandosi dei consigli, a volte può essere molto positivo, anche se non dobbiamo mai dimenticarci che il nefrologo è il nostro primo riferimento. Condividere la sensazione, chiedendo un consiglio, è importante, ma non bisogna mai trascendere nell'automedicazione, perché rischiamo di creare a noi stessi, talvolta, grossi problemi.

L.: La "I" di AIRP con voi ha avuto un senso molto preciso perché questa "I" vuol dire "Italiana - Associazione Italiana"; ecco, a vostro parere, c'è un'unione, c'è dialogo fra le nefrologie italiane?

T.: A tutta la comunità scientifica noi vogliamo dire "grazie". Nella nostra fattispecie, il Policlinico di Bari ci ha sostenuto in alcune fasi di questo percorso, il Policlinico di Padova ci ha portato a concretizzare il trapianto, che avevamo cominciato a pianificare circa un anno e mezzo fa, e quindi è bellissimo quando non ci sono frontiere, non ci sono confini, non ci sono insegne. Noti che quel famoso "giuramento di Ippocrate" viene portato avanti, da chi indossa quel camice, con amore, competenza e professionalità, senza puntare alla mera visibilità, e permette a delle persone di rinascere. lo di questo sono infinitamente grata, perché la nostra esperienza è stata più che positiva in questo senso, c'è stata una vera unione tra i due Istituti, per competenze e intenti, un vero sodalizio, una "multidisciplinarietà".

V., T.: C'è stato un incontro tra professionalità e il Prof. Gesualdo e la Dott.ssa Guido hanno collaborato fattivamente e proficuamente con il Prof. Rigotti e l'intera sua equipe, la Prof.ssa Furian, la Dott.ssa Silvestre e la Dott.ssa Neri, permettendoci di realizzare il nostro sogno e di superare tutti gli ostacoli che sulla strada abbiamo incontrato. Credo che, in questo caso, sia doveroso fare i nomi, affinché non si parli in astratto, per dare merito a questi specialisti, e in prima battuta "persone" che hanno messo tutta la loro umanità oltre che competenza a favore di questo progetto di rinascita. 


\section{L.: Tania, parliamo da donna a donna. Ma tu dov'eri quel giorno? Quando hai potuto dire: "Sì, si può fare"?}

T.: È stato dopo Natale dello scorso dicembre, il nostro è stato un percorso a ostacoli, perché, fino all'ultimo, come ti dicevo anche giorni fa al telefono, non si era più sicuri se io potessi donare a Vittorio, e questa cosa, devo dire, poteva essere anche vissuta come un "fallimento", perché ci avevamo investito, al di là del tempo, aspettative ed emozioni. Dopo Natale, il Prof. Gesualdo scrisse un messaggio a Vittorio non appena ebbe l'esito dell'ultimo mio esame di biopsia ai reni e scrisse: "Il rene è normale, Tania è leggibile e, quindi, adesso avviserò subito Padova". Questa cosa che abbiano comunicato tra di loro è stato per noi un alleggerimento ulteriore, perché, in quel momento per noi così carico emotivamente, forse non saremmo riusciti ad avere un ruolo attivo, perché sei talmente emozionato che magari non hai la lucidità sufficiente. Dov'ero io quando è arrivato il messaggio dell'esito finale? lo ero in terrazza, stavo lavorando, e Vittorio venne a dirmelo, ci siamo guardati e abbiamo pianto, perché ancora non era avvenuto niente ma sapevamo che era un nuovo inizio.

\section{L.: Vittorio, invece, per te, che prospettiva ti sei visto davanti?}

V.: La prospettiva, come sempre succede in queste situazioni, ti offre un mix di emozioni: da un lato c'è l'eccitazione di veder compiuto un percorso iniziato tanto tempo prima e che sogni da una vita, ma che, fino all'ultimo, magari avevi pensato di dover abbandonare, dall'altro tanta paura, perché, ovviamente, non sai mai cosa andrai ad affrontare e realizzi che quello che hai immaginato sta accadendo veramente e il tempo per pensare a tutto ciò è stato breve: immagina, ho ricevuto la comunicazione il 27 dicembre alle sette di sera e il 28 mattina alle 8.00 sono stato già contattato dall'Ospedale di Padova, che voleva fissarmi la data della nefrectomia pre-trapianto per il 2 gennaio. Quindi, di conseguenza, da un lato è stato un bene che ci sia stato poco tempo per elaborare questa informazione, dall'altro lato siamo stati catapultati in questa nuova situazione alla velocità della luce.

\section{L.: La notte di San Silvestro voi dove eravate? Che cosa avete pensato in quella notte?}

T.: Festeggiavamo il Capodanno guardando all'anno che arrivava con una nuova prospettiva, soprattutto con la speranza che il Covid-19 in quel momento non si abbattesse su di noi, visto che già c'è il rischio che ogni intervento porta con sé e non hai alcuna garanzia sull'esito, ma sai di aver fatto tutto quello che avresti potuto fare, ti senti nel posto giusto, nelle mani giuste e di aver preso parte al gioco della vita.

\section{L.: Ma, in quel momento della partenza, chi di voi era più agitato, più apprensivo? Chi dei due?}

V.: Sicuramente lo. lo dei due sono la persona più apprensiva in generale. Immaginatevi nel momento in cui ti vai ad approcciare a qualcosa del genere, che rappresentava per me un grande cambiamento e che avrebbe significato tanto anche per Tania. Quindi, unite un po' il mio essere già un po' più ansioso di norma con questo tipo di situazione: è chiaro che, tra i due, il più ansioso ero io. Una delle sfide che abbiamo vissuto nel momento in cui ho chiuso il telefono è stato organizzare la partenza in tempi rapidi, così ho guardato Tania negli occhi e le ho detto: "Adesso come ci andiamo a Padova?". Abbiamo provato a pensare a tutto, con i pro e i contro: la macchina, il treno, l'aereo. Per fortuna, poi siamo riusciti a trovare un aereo che ci avrebbe portato a Padova via Roma, dal momento che, in questi tempi di Covid-19, la gran parte dei voli è sospesa. Ovviamente, un viaggio all'insegna della precauzione: doppie mascherine, lavaggio di mani ogni 10 minuti.

T.: Io ho organizzato i tamponi, perché Padova ci chiedeva, in questo momento specifico, di arrivare tamponati.

\section{L.: Arrivati a Padova il primo pensiero qual è stato?}

V.: In realtà, a Padova ci siamo stati per ben due volte a gennaio, la prima per un intervento di nefrectomia e laparocele (che dovevo riparare per la sesta volta) e la seconda, dopo 25 giorni, per il trapianto. II primo ricordo che mi viene in mente è quando ho salutato Tania, prima di entrare in reparto, per effettuare il primo intervento di preparazione alla ricezione, in un'area dove i visitatori dovevano fermarsi. Salutarsi, sapendo di rivedersi fisicamente solamente alla fine di un intervento chirurgico, è chiaramente un'emozione molto forte, perché ti saluti cercando di infondere sicurezza alla persona che hai di fronte, ma, allo stesso tempo, traspare tutta la fragilità e la paura che era comunque presente nei suoi e nei miei occhi. 
T.: Già. Un altro ricordo carico di emozione che mi viene in mente è quando ero già mezza moribonda sotto anestesia in sala operatoria e mi dissero sorridendo: "Signora, neanche oggi ha un po' di ansia? Speravamo che le venisse almeno un po', dal momento che anche oggi ha la pressione bassa 90/65, ma non si preoccupi ci penseremo noi a tenerla alta". $\mathrm{E}$ io seraficamente risposi: "Perché devo essere in ansia? Sono nel posto giusto, con le persone giuste, per la persona giusta".

L.: Quando vi siete rivisti, dopo che eravate stati costretti a separarvi, perché uno doveva andare da una parte e l'altro doveva andare dall'altra ma tutti e due con lo stesso fine, cosa vi siete detti? Immagino che sarà stato un momento molto emozionante.

T.: Il momento dell'incontro è stato un po' come incontrarsi sull'altare e, per me, è avvenuto in due momenti. II primo coincide con la mia uscita dalla sala operatoria e il suo ingresso. lo pensavo di viverlo più pienamente quell'incontro, in maniera cosciente, ma non avevo messo in conto che uscivo dopo un intervento di alcune ore e sotto anestesia, quindi l'unica cosa "intelligente" che mi sono trovata a dirgli, invece di tante cose che mi ero programmata di dire, è stato: "Mi raccomando", come se l'esito dell'intervento dipendesse da lui, cara Luisa, e lui mi ha risposto: "Faccio del mio meglio".

II secondo momento, in cui ricordo l'intensità del nostro incontro, è stato nel post-intervento. Avevo paura di non beccarlo alla sua uscita dalla sala operatoria, prima di andare nella semi-intensiva, avevo paura di dormire, in quel momento, e li, molto carinamente, tutti gli infermieri e i medici mi hanno detto: "Tania stai serena, ti svegliamo noi per questa occasione, dormirai dopo, non ti preoccupare che, quando Vittorio passa, lo sguardo ve lo facciamo incrociare".

V.: Hanno fatto di più, perché io ero nel letto, hanno fatto entrare il mio letto nella stanza di Tania per permetterci di guardarci negli occhi e, ovviamente, lei era ancora un po' sonnolenta, io anche, e mi ricordo di aver celebrato la quantità di urina che avevo fatto durante l'operazione. Era così tanto il desiderio di condividere il successo che ho condiviso un dettaglio che magari non era particolarmente "romantico", ma per noi significava: "È andato tutto bene, sto bene, il rene funziona". Pensa che la prima notte dopo il trapianto ho urinato quasi 10 litri, che, per l'Ospedale, è quasi un record, nel senso che è difficile che un rene, specialmente di una persona non compatibile, parta con questo sprint, secondo me era figlio del tanto desiderio che avevamo di questa cosa e forse anche dell'entusiasmo di Tania, che mi ha passato con la donazione del suo rene.
T.: lo ci credo a questo trasferimento di entusiasmo, in fondo è pur sempre una parte di me che è dentro di lui e che, io mi auguro, possa restare per sempre. In fondo, Vittorio, con le sue parole, ci ha come riportato l'attenzione sull'avvenuta ri-nascita e, come accade con la nascita di un bambino, quando il neonato piange è un buon segno!

\section{L.: Vittorio ti ricordi il mio sms?}

V.: Mi ricordo assolutamente. Mi ricordo il tuo messaggio, in cui mi chiedevi: "Come stai?", che è una frase semplice ma che raccoglieva il desiderio di condivisione. Mi ricordo che, il primo giorno dopo il trapianto, lo abbiamo passato scambiandoci continui messaggi, dettagli, sensazioni, ed è stato di grande sollievo per me, e lo stesso vale per tutti i messaggi d'affetto ricevuti da tanti soci AIRP.

L.: Quando si vive un'esperienza così forte, così emozionante non si riesce a stare soli. In camera voi chi avevate?

V.: La cosa bella è che, in quella stessa settimana, AIRP aveva una delegazione all'interno del Centro Trapianti, perché un'altra socia, Giulia Russo, ha effettuato anche lei un trapianto da vivente.

T.: Suor Giulia era nella mia stanza. Giulia fa parte del gruppo di AIRP, una suora che ha tante vite in una, fa missione in Africa da oltre 35 anni, ognuno in stanza partecipava alla vita dell'altro, diventando, così, una seconda famiglia. Con le persone della mia stanza abbiamo creato anche un gruppo su WhatsApp, che abbiamo chiamato "Le frittelle", perché la domanda che avevo fatto a Elisa, una ragazza trapiantata della stanza, è stata: "Elisa cosa farai per prima cosa dopo il trapianto?" e lei: "Finalmente mangerò le frittelle!".

L.: Vittorio, subito dopo il trapianto, quando sei rientrato in camera, qual è stato il tuo primo desiderio? Cosa avresti voluto fare per prima cosa?

V.: Il cibo è stato anche per noi in stanza un continuo argomento di discussione, perché, nella vita di un dializzato o di una

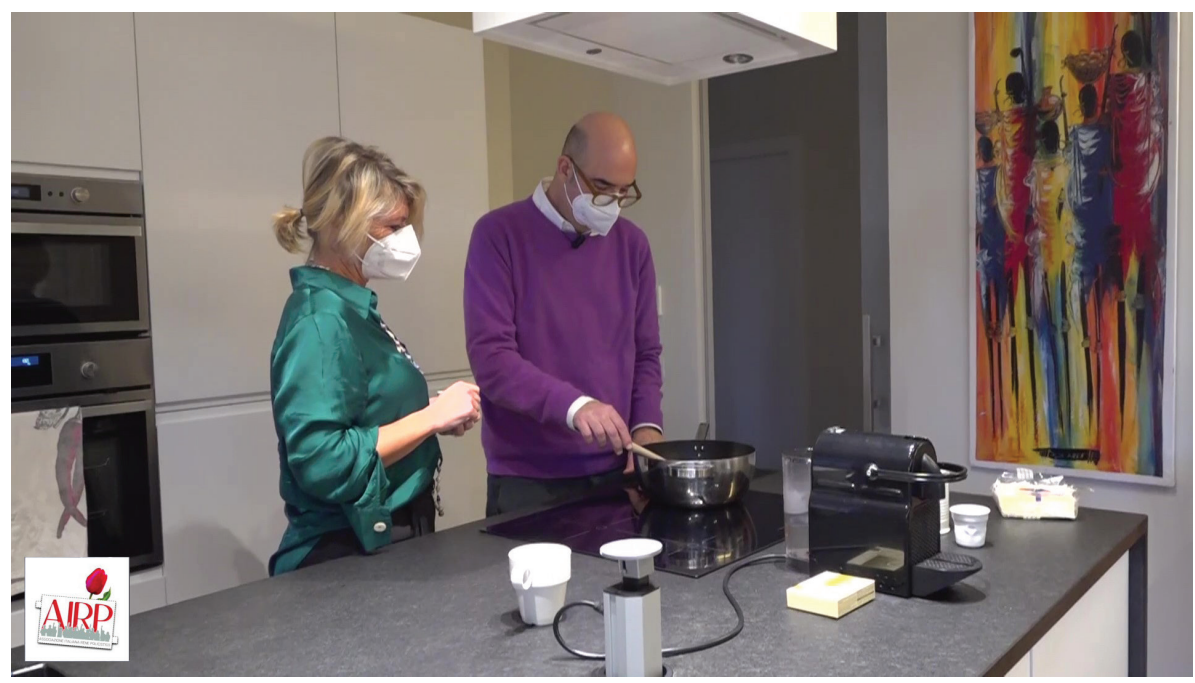




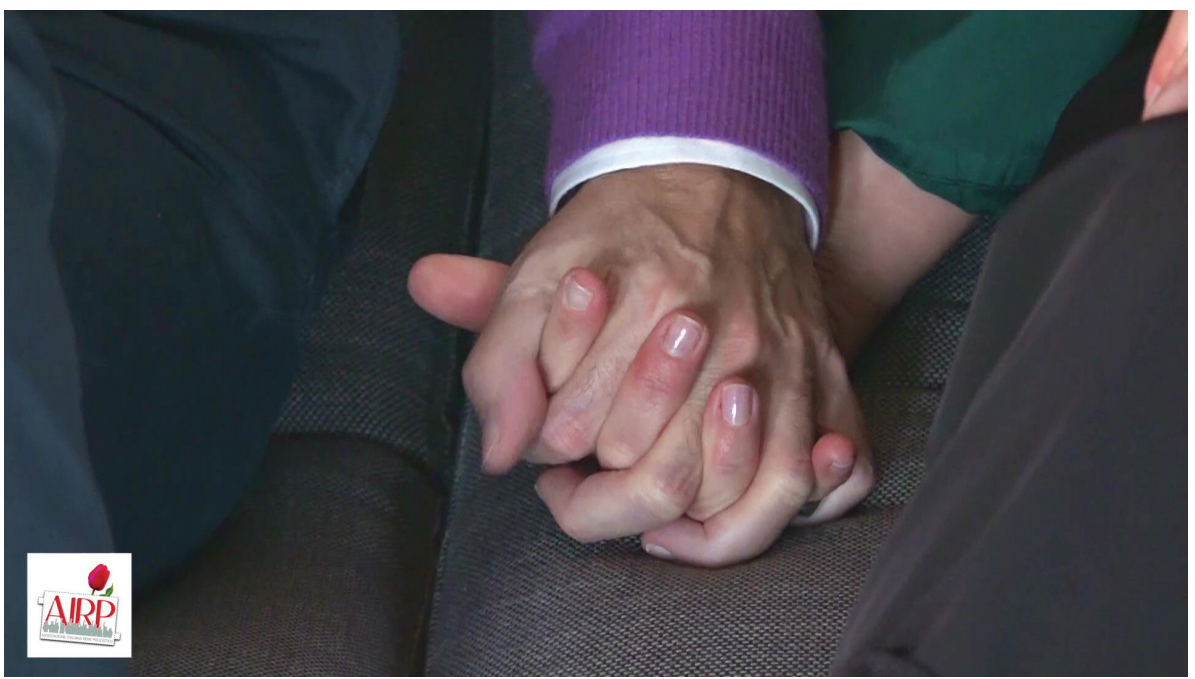

V.: È vero, il primo incontro è avvenuto quando Tania è riuscita ad alzarsi dal letto dopo un paio di giorni e venne da me e mi ricordo che ci guardammo negli occhi, e la prima cosa che abbiamo fatto è stato piangere e, anche se non ci si poteva abbracciare, c'era una fisicità spirituale molto forte, un'unione molto forte e, quindi, questo è stato il primo incontro. II secondo incontro è stato sulla porta del reparto, io sono potuto uscire dopo 10 giorni dall'intervento. Non sapevamo cosa fare, se potevamo abbassare le mascherine e darci un bacio, abbracciarci, quindi diciamo che, in termini di fisicità, è stato molto complicato. A un certo punto, la mandai a

persona con rene policistico con insufficienza renale terminale, l'alimentazione è cruciale. Abbiamo dovuto fare per tanti anni rinunce e sacrifici e, quindi, la discussione anche nella nostra stanza di trapiantati era "la prima cosa che mangerai quando esci" e mi ricordo che il mio vicino di letto mi diceva: "lo mangerò 3 banane, quando esco da qua, me le faccio portare da mia moglie quando viene a prendermi". E, invece, il mio unico desiderio era di mangiare una pizza. Pensa quanto queste semplici cose, che fanno parte della vita di ognuno, per tante persone rappresentano ancora oggi un desiderio fortissimo.

L.: Voi, che avete vissuto pienamente questa esperienza, che messaggio volete lanciare alle persone che non sono ancora bene informate, che non sanno che si può anche donare un rene da vivente?

V.: Raccogliere informazioni dalle persone giuste, perché oggi il web è pieno di fake news, di storie fatte solo per attirare like dove si parla di cose fantomatiche. Oggi, la donazione da vivente permette di poter dare una nuova vita a qualcuno e anche l'altro tipo di donazione permette di dare un senso, se mai un senso ce lo possa avere, alla morte di una persona, perché questa persona continua a vivere dentro il corpo di qualcun altro e questa è una cosa che permetterebbe all'Italia di avere delle liste di attesa meno lunghe e che consentirebbe, quindi, a chiunque di aspettare meno tempo, di vivere una vita più etica, più civile $\mathrm{e}$, quindi, permetterebbe a tante persone, che in questo momento stanno soffrendo, di tornare a vivere come lo sto facendo io.

\section{L.: In un periodo in cui la fisicità è interdetta e non ci si può abbracciare, quando vi siete visti voi quell'abbraccio ve lo siete dati?}

V.: Mi ricordo che Tania mi è venuta a prendere sulla porta del reparto, quando sono uscito dall'ospedale.

T.: No, scusa, Vittorio, ti sei perso il momento in cui ti sono venuta a trovare con la pancera nella semi-intensiva. Un momento celebrativo. fare un tampone a Padova e le dissi: “Ok, fai un tampone, perché così possiamo finalmente darci un bacio e un bacio penso che ce lo siamo meritato".

T.: Sì, è stato tutto così strano, perché la prima cosa che cerchi è un contatto e vuoi quasi perderti in un abbraccio che ti riconsegni tutto lo sforzo che hai fatto. Me lo immaginavo come il più stretto di sempre e, invece, lui era delicato, fragile, da proteggere. I medici mi hanno detto: "Ha il sistema immunitario azzerato, mi raccomando". Io chiesi proprio delle cose basilari ai medici, pur vergognandomi quasi, perché mi sentivo come un'adolescente alle prime armi con il primo amore. Chiesi loro: "Ma lo potrò baciare?" e i medici mi guardarono e, per rassicurarmi, mi dissero: "Signora, se evitate, in questo periodo, è meglio". Poi, quando Vittorio uscì dall'ospedale, mi disse sorridendo: "Se ci vogliamo dare un bacio, e credo che ce lo siamo anche meritato, vai a tamponarti".

\section{L.: Tania come stai, come si vive con un rene solo?}

T.: lo non so ancora come si vive perché è solo un mese che sono uscita da questo intervento e, in quanto il mio primo, devo dire la verità, ho anche faticato a uscirne. È da tre giorni che mi sento finalmente in pieno possesso del mio corpo, ho visto il mio viso riprendere delle fattezze di un viso in salute, cioè con le stesse rughe di prima e non di più. Non mi riconoscevo inizialmente, lui non si riconosceva per altri versi, poiché è molto dimagrito, e anch'io sono dovuta scendere a patti con la sua nuova fisicità, di certo anche più armoniosa di prima, perché aveva una pancia grossa per via dei grossi reni policistici. Ho conosciuto, grazie a questa storia, perché ogni storia ti spinge a condividere ciò che stai vivendo con altre persone, tante altre storie, anche di persone che conoscevo da tempo ma con cui magari non sono mai entrata in questa intimità, che mi dicevano: "Sai, Tania, io sono monorene da 20 anni", e io: "Ma come, non me l'hai mai detto" e, di risposta, ricevevo un: "Beh, non sono cose che si dicono". E, così, è accaduta 
questa alchemica e profonda connessione umana con tante altre persone che ci sono state vicine in questo viaggio, che, per rassicurarmi, hanno voluto aprirmi il loro cuore, ed io dico a me stessa: "Quanto non si sa delle persone che già ci conoscono".

\section{L.: E tu, Vittorio, la tua ripresa fisica e psicologica come va?}

V.: Grazie per la domanda, perché, secondo me, questo aspetto è uno tra quelli più importanti da condividere: la prima ripresa non è tanto quella fisica quanto quella psicologica, perché chi ha vissuto per tanti anni con privazioni e rinunce oggi vive una situazione di cambiamento mentale, perché deve abituarsi a pensare di non essere più "malato", ed è qualcosa da cui ci stacchiamo con grande difficoltà.

Quindi, di conseguenza, l'inizio è complicato, perché i medici ti dicono che "puoi mangiare tutto" però, in realtà, nel tuo retrocranio continui a limitarti nel mangiare alcuni alimenti come la frutta e la verdura, perché la tua testa crede che tu sia ancora una persona affetta da rene policistico.

II secondo ostacolo è abituarsi a una nuova fisicità. Avendo rimosso entrambi i reni, che pesavano complessivamente 12 chili, e avendo perso altri 8 chili nella prima settimana post-trapianto, perché, probabilmente, avevo tanti liquidi nel corpo che il "super" rene di Tania ha permesso di poter eliminare, dal $1^{\circ}$ gennaio ho perso quasi 20 chili e, di conseguenza, questo mi ha portato comunque uno stato generale di anemia, un po' di stanchezza da cui piano piano sto recuperando. Guardarsi allo specchio, poi, è complesso, perché, a volte, non ci si riconosce, soprattutto quando si osserva l'addome e l'altro curioso aspetto da considerare è che devi rifare l'armadio perché ti va tutto grande. L'aspetto positivo, in questo caso, è che ho un armadio molto grande e che conservo ancora le cose che indossavo 20 anni fa e che sto riesumando piano piano, però non vedo l'ora di poter mettere il naso fuori casa e fare una buona seduta di shopping.

Quindi, oggi, al di là di chi ha avuto la fortuna come me di avere al suo fianco un angelo che ha donato un rene e di chi è magari in lista d'attesa, il mio consiglio è di continuare a vivere con ottimismo, di tenersi informato su quello che oggi la scienza ci propone, perché di soluzioni ce ne sono tante e ce ne saranno tante in futuro, di avere una relazione costante con il proprio nefrologo e di controllarsi spesso con piena coscienza di quello che non si può fare e vivendo con felicità quello che, invece, si può fare.

L.: Senti, Vittorio, ma, alla fine, la pizza l'hai mangiata o no? Un'altra curiosità, che pizza hai ordinato?

V.: In realtà l'abbiamo ordinata il giorno che siamo tornati a Bari e, alla fine, è stata una "semplice" pizza margherita, sempre per via di quel retropensiero da "ammalato" che fa fatica ad allontanarsi, mentre, in cuor mio, l'avrei voluta, invece, piena di verdure e di cose prima "proibite".

L.: Tania e Vittorio, io vi ringrazio veramente di tutto cuore per questa vostra testimonianza. È una testimonianza che sarà molto molto utile a tutti $i$ nostri pazienti e alle famiglie dei nostri pazienti. lo vorrei ripromettermi, però, di rivederci. Voi siete stati molto ospitali, ma io vorrei tanto rivederci in un momento in cui potremo andare a mangiare fuori o potremo cucinarci, io vi potrò fare sicuramente un risotto con gli ossibuchi e voi mi offrirete qualcos'altro, vedremo cosa cucinare insieme.

V.: Per quanto riguarda il menù ci possiamo organizzare. Noi abbiamo vissuto 15 anni a Milano e siamo fan delle cotolette a "orecchio d'elefante", quindi, se tu ce lo permetti, verremo noi a visitarti e, magari, ci offrirai un buon risotto con "I'òs büüs" e una cotoletta alla milanese, mentre, quando ci ricambierai la visita a Bari, ti farò mangiare, ma solo a te, il nostro pesce crudo, per cui ovviamente a Bari siamo famosi.

T.: E io ti accompagno, Luisa, perché alla fine credo di aver fatto già la mia parte, ora non è che devo vivere sacrificata. Vittorio non sarà altro che felice per noi mentre mangiamo questo pesce crudo, mentre lui si mangerà altro, magari una frittura di calamari, e sarà contento di quello che mangia.

\section{L.: Vi abbraccio veramente con tutto il cuore, ragazzi, e continuiamo sempre con I'hashtag \#AIRPerLaVita! \\ V., T.: Viva I'AIRP!}

Intervista raccolta da:

Luisa Sternfeld Pavia,

Presidente AIRP - Associazione Italiana Rene Policistico

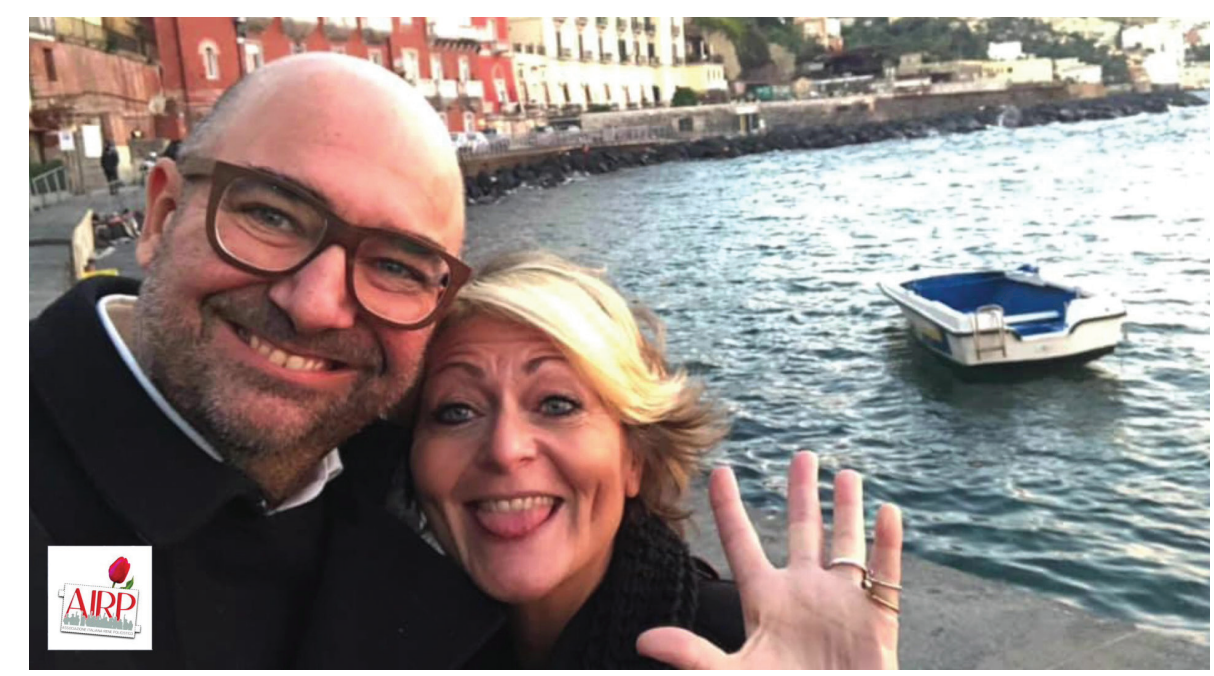

